\title{
A personal retrospective on the 60th anniversary of the journal biological cybernetics
}

\author{
Henry D. I. Abarbanel ${ }^{1}$ \\ Published online: 26 May 2021 \\ (c) The Author(s), under exclusive licence to Springer-Verlag GmbH Germany, part of Springer Nature 2021
}

A long time ago in a galaxy far, far away..., maybe circa 1995:

While sitting quietly in my office at the Institute for Nonlinear Science at UCSD, a colleague whom I did not know well, Allen Selverston, appeared at my door and presented to me a drawing of the Pyloric Central Pattern Generator (CPG) depicted on a paper napkin.

He stated that he knew the circuit diagram of this rhythmic functional neural circuit and could identify and measure the electrophysiological properties of the neurons on any given day. He came to ask me for help in understanding how it worked.

This initiated a long collaboration with Selverston that started by biweekly meetings in his laboratory and our offices (we did not have a real laboratory until he moved in and established one a few years later). We met in his laboratory where he introduced us to how neurons work, and then two weeks later he and his group appeared in our conference room to hear how a bunch of physicists saw the nonlinear electrical oscillator he called a neuron.

This exchange, not really hampered by our training in quite different scientific cultures, went very well.

Two years passed and we were invited to a Nonlinear Dynamics conference in Bonn, Germany, and Selverston was invited to a CPG meeting in Eilat, Israel. We agreed that Selverston would go to Bonn and present a talk on our joint work, and one of us would be there in the room to help with

To highlight the scientific impact of our Journal over the last decades, we asked authors of highly influential papers to reflect on the history of their study, the long-term effect it had, and future perspectives of their research. We trust the reader will enjoy these first-person accounts of the history of big ideas in Biological Cybernetics.

Henry D. I. Abarbanel

habarbanel@ucsd.edu

1 Physics and Scripps Institution of Oceanography, University of California San Diego, San Diego, USA any questions. We also agreed that I would go to Eilat and present a talk on our joint work, and $\mathrm{Al}$ would be there in the room to help with any questions. A nice symmetry we all thought. The idea was that if one side of us could explain our joint work to colleagues on the other side, it might generate a new set of collaborations among neurobiologists and physicists. These many years later, collaborations such as we had envisioned are part of academia writ large. Alas, we can hardly take credit for that very satisfying outcome.

A few years later we had a visitor, Martin Falcke, now at the Max Delbrück Center for Molecular Medicine, then already an established scientist in the role of Calcium neural dynamics. We jointly worked on the function of Calcium in a Pyloric CPG. This work, and here enters the connection of this rambling essay to Biological Cybernetics, was published as Martin Falcke, Ram'on Huerta, Mikhail I. Rabinovich, Henry D. I. Abarbanel, Robert C. Elson, and Allen I. Selverston, "Modeling Observed Chaotic Oscillations in Bursting Neurons: The Role of Calcium Dynamics and $\mathrm{IP}_{3}$," Biological Cybernetics, 82, 517-527 (2000).

To be honest, I had not really heard much of this journal, but I certainly had heard of the famous mathematician at MIT, Norbert Wiener, who was one of the founders of this journal. In 1948, he published the book "Cybernetics: Or Control and Communication in the Animal and the Machine".

Twelve years later, he participated in founding this journal, along with the leadership of Werner Reichhardt, called at the time Kybernetik. It was described as "A Journal Dealing with the Transmission and Processing of Information as well as with Control Processes in both Animals and Automata."

To quote a future editor-in-chief of this journal, Leo van Hemmen,

And, if I may say so, like Wiener, one of the founding fathers of Biological Cybernetics, I have always seen the journal as one that, on a solid mathematical basis, ought to disseminate the kind of mathematical 
understanding that is needed to quantify the plethora of fascinating phenomena in neuroscience.

Leo will appear prominently later in this retrospective.

Going on Wiener's eminent reputation (he passed away in 1964 at age 70), I went in with serious biological scientists to publish the paper with Falcke in this journal. That conjunction of events led my colleagues and myself to publish a few papers in Biological Cybernetics over the years. It had become clear to us that it was almost the only venue where, what we now call Neural Computation, was practiced at a high level.

After a gap of about a decade, I again encountered Leo, now the editor-in-chief of Biological Cybernetics (2006-2017).

I had turned to the study of the dynamics of avian song production and control along with Daniel Margoliash (University of Chicago), Gabriel Mindlin (University of Buenos Aires), and Richard Hahnloser (ETH, Zurich) as guides and scientific collaborators. In March 2010 at the annual large meeting of the American Physical Society, that year in Portland, Oregon, Dezhe Jin from Pennsylvania State University and now associated with the Penn State Department of Physics and Center for Neural Engineering, invited Margoliash, Hahnloser, and myself to speak at a special session devoted to the dynamics of avian song.

Jin had been a graduate student at UCSD studying plasma Physics, and he invited me because he, apparently, had fond memories of UCSD. At Jin's session, all of us gave our talks about our work on the designated topic, and at the conclusion, we discovered Leo van Hemmen in the audience.

After the formal talks, Leo approached me, expressed an interest in our work, and then invited us to publish 1000 pages in Biological Cybernetics. I was, of course, flattered by his interest, and I was totally taken by surprise by his suggestion. I assured him that I was certain I did not have, and surely would not have, 1000 pages of scientific work for his, or any journal. Leo, as was his wont, persisted. We struck a deal to give it a try, and I headed back to La Jolla, while he went home to the Technical University of Munich.
I must acknowledge that we spectacularly failed in our endeavor to meet Leo's goal.

We did publish many papers over the next few years in this journal, but maybe used $10-15 \%$ of our designated allotment.

Leo, recognizing that I had disappointed him, asked me in 2014 to become an Associate editor of this journal. Once again I protested that I was a terrible editor, and he surely could find many better persons to help out. Leo compromised by promising to only send me a "few" papers in any year to review. He kept his word, but also sent me many papers from UCSD colleagues. I had to invoke a conflict of interest, not Leo's worry, to avoid reviewing those submissions.

Over the following years, this journal added to its title the words: Advances in Computational Neuroscience and in Control and Information Theory for Biological Systems. I applauded that expanded view of the journal's worldview, and I continue to do so. I also am proud to be associated with the continuing leadership of Biological Cybernetics: Editorin-Chief: Benjamin Lindner and the Co-Editors-in-Chief: Peter Thomas, Jean-Marc Fellous, and Paul Tiesinga. They, along with the other members of the Board of Associate editors, have seen this 60th anniversary of the journal as a time to renew the vision of Wiener from 1961 and adapt it to the twenty-first century. Reichhardt and Wiener would be quite pleased with their creation's posture in the quantitative scientific understanding of neuroscience.

A final word: Peter Thomas asked me to try my hand at this essay earlier this year. Thank you, Peter.

Publisher's Note Springer Nature remains neutral with regard to jurisdictional claims in published maps and institutional affiliations. 